\title{
Fatores associados ao uso inapropriado do pronto atendimento
}

\author{
Factors associated to inappropriate use of emergency services
}

Tatiane Baratieri (https://orcid.org/0000-0002-0270-6395) ${ }^{1}$

Maicon Henrique Lentsck (https://orcid.org/0000-0002-8912-8902) ${ }^{2}$

Ligiana Pires Corona (https://orcid.org/0000-0001-5298-7714) ${ }^{3}$

Keroley Paes de Almeida (https://orcid.org/0000-0001-7560-4640) ${ }^{4}$

Ana Cláudia Garabeli Cavalli Kluthcovsky (https://orcid.org/0000-0002-4772-2970) ${ }^{5}$

Sônia Natal (https://orcid.org/0000-0001-6155-4785) ${ }^{6}$

\footnotetext{
${ }^{1}$ Departamento de Enfermagem, Universidade Estadual do Centro-Oeste. Rua Alameda Élio Antonio Dalla Vecchia 838. Vila Carli. 85040-167 Guarapuava PR

Brasil.

baratieri.tatiane@gmail.com

${ }^{2}$ Departamento de

Enfermagem, Universidade

Estadual do Centro-Oeste.

Guarapuava PR Brasil.

${ }^{3}$ Faculdade de Ciências

Aplicadas, Universidade Estadual de Campinas. Campinas SP Brasil.

${ }^{4}$ Departamento de

Enfermagem. Universidade Estadual do Centro-Oeste.

Guarapuava PR Brasil.

${ }^{5}$ Departamento de

Medicina, Universidade

Estadual de Ponta Grossa.

Ponta Grossa PR Brasil.

${ }^{6}$ Departamento de Saúde

Pública, Universidade

Federal de Santa Catarina.

Florianópolis SC Brasil.
}

\begin{abstract}
The increase in the demand for Emergency Services is a worldwide phenomenon, and its inappropriate use is one of the main problems. Factors related to the inappropriate use must be investigated to guide improvement in the quality of services. This article aims at analyzing factors associated with the inappropriate use of emergency services. This is a cross-sectional study with secondary data from 384 medical records from two emergency services in a city in central-southern region of the state of Paraná, Brazil, in 2013. A descriptive, bivariate using Pearson's chi-squared test was performed to verify associations between inappropriate use and independent variables. Multiple Logistic Regression was also performed. The proportion of inappropriate use of Emergency Services was $73.4 \%$ and was associated with people with some primary health care sensitive conditions, residents in areas without primary care coverage, people between 0 and 11 years old, and 12 to 17 years old, care in the first trimester of the year, respiratory diseases, lesions and poisonings and diseases of the musculoskeletal system. Primary care must be strengthened to reduce the inappropriate use of care, especially in the attention to primary health care sensitive conditions and to increase the socio-organizational accessibility.

Key words Emergency medical services, Primary health care, Health evaluation
\end{abstract}

Resumo $O$ aumento da demanda pelo pronto atendimento é um fenômeno mundial, e seu uso inapropriado é um dos principais problemas. Há necessidade de identificar os fatores relacionados para orientar a melhoria da qualidade dos serviços. O objetivo deste artigo é analisar os fatores associados ao uso inapropriado em serviços de Pronto Atendimento. Estudo transversal com dados de 384 prontuários de pacientes atendidos em dois serviços de pronto atendimento de um município do Centro-Sul do Paraná, Brasil, em 2013. A análise foi descritiva, bivariada pelo Qui-quadrado de Pearson para verificar associações entre uso inapropriado e variáveis independentes. Realizou-se Regressão Logística Múltipla. A proporção do uso inapropriado de pronto atendimento foi de $73,4 \%$ e apresentou associação com pessoas com alguma condição sensivel à atenção primária, residentes em áreas sem cobertura desta atenção, pessoas entre 0 e 11 anos, e 12 a 17 anos, atendimento no primeiro trimestre do ano, doenças do aparelho respiratório, lesões e envenenamentos e doenças do sistema osteomuscular. Necessita-se fortalecer a atenção primária para redução do uso inapropriado do pronto atendimento, especialmente na atenção às condições sensivieis à atenção primária e ampliação da acessibilidade sócio-organizacional. Palavras-chave Serviços médicos de emergência, Atenção Primária à Saúde, Avaliação em Saúde 


\section{Introdução}

O aumento pela busca de pronto atendimento (PA) pode ser considerado um fenômeno mundial. Quando o serviço é dispensado aos usuários com condições clínicas não urgentes ou emergentes $^{1-5}$, caracteriza-se o uso inapropriado dos serviços de urgência/emergência.

$\mathrm{O}$ atendimento de urgência/emergência tem por objetivo realizar procedimentos para estabilizar, de forma imediata, condições clínicas específicas, mas não de forma continuada ${ }^{6}$. No entanto, as pessoas frequentemente procuram o PA para obter atenção imediata, independentemente de ser uma situação aguda ou crônica agudizada, o que acarreta problemas para a provisão desses ${ }^{6}$. Evidências demonstram que o acesso ao PA por uso inapropriado varia de $24 \%$ a $40 \%$, com picos de até $90 \%$ de todo o atendimento ${ }^{2,4,6}$.

Em países como os Estados Unidos, Canadá, Reino Unido e Austrália a demanda por serviços de emergência aumentou entre 3\% a $6 \%$ ao ano 7 . Sua consequência é a superlotação dos serviços e maior tempo de espera ${ }^{7,8}$, aumento dos custos para o sistema de saúde ${ }^{2}$, insatisfação dos usuários $^{8}$ e precarização do atendimento para os casos urgentes ${ }^{4,8}$.

No Brasil, a escassez de estudos publicados sobre o uso inapropriado em serviços de PA devido à falta de informação sobre o uso dos serviços de emergência hospitalares e das Unidades de Pronto Atendimentos (UPA), impacta na organização e funcionamento da rede dos serviços de urgência e emergência, assim como nos resultados do cuidado. $\mathrm{E}$ a análise dos motivos que levam os usuários a procurar o PA é importante para identificar problemas dessa rede.

As UPAs, com serviços 24 horas, devem, prioritariamente acolher os usuários e familiares em situação de urgência e emergência, sempre que buscarem atendimento; articular-se com os demais pontos de atenção e com os serviços de apoio diagnóstico e terapêutico; prestar atendimento resolutivo e qualificado aos usuários acometidos por quadros agudos ou agudizados de natureza clínica, cirúrgica e de trauma; prestar apoio diagnóstico e terapêutico e encaminhar para outros serviços quando necessário ${ }^{9}$. Por tratar-se de uma estrutura de complexidade intermediária entre os serviços de Atenção Primária à Saúde (APS) e hospitalar, a observação do seu uso pode subsidiar o aperfeiçoamento dos fluxos de atendimento.

A população pode optar pelo atendimento de urgência e emergência por diversos motivos, os quais estão relacionados a maior acessibilidade socio-organizacional (horário de funcionamento, tempo de espera e disponibilidade de profissionais, especialmente o médico) comparado a Atenção Primária à Saúde (APS), falta de confiança na APS; percepção de serviços baseados na emergência como necessários; opiniões de familiares/amigos/profissionais de saúde; proximidade ao PA; agilidade no encaminhamento para consulta com especialistas e realização de exames, dificuldade de agendamento para atendimento na APS, conveniência para o usuário ou falta de conhecimento sobre o fluxo de atendimento nos diferentes pontos de atenção à saúde $\mathrm{e}^{7,10,11}$, problemas na estruturação da rede assistencial, na coordenação e integralidade do cuidado ${ }^{12}$.

Além disso, essa análise pode ser feita por meio de indicadores de saúde, como as Condições Sensíveis à Atenção Primária (CSAP), as quais são comumente utilizadas como indicador de atenção hospitalar e caracterizam-se por um grupo de causas e diagnósticos que podem ser manejadas oportunamente na $\mathrm{APS}^{13}$, sem necessidade de atendimento em serviços de PA, exceto em situações de agudização, que se caracterizam como urgência e emergência.

Embora o uso do PA possa parecer apropriado do ponto de vista do paciente, dadas as limitações existentes em outros níveis dos cuidados de saúde, este tipo de utilização aumenta a demanda por cuidados que poderiam ser mais bem gerenciados em outros níveis e que em certo sentido concorre com verdadeiros casos de emergência ${ }^{6}$. Portanto, este estudo tem por objetivo analisar fatores associados ao uso inapropriado em serviços de PA.

\section{Materiais e métodos}

Estudo transversal que utilizou dados contidos em prontuários de pacientes atendidos em dois serviços de PA em um município do Centro-Sul do Paraná, no período de 1 de janeiro a 31 de dezembro de 2013.

Os serviços de urgência e emergência do município são vinculados à Rede de Urgência e Emergência da região de saúde, oferecem atendimento 24 horas, ininterrupto, para usuários de todas as idades, e utilizam o acolhimento com classificação de risco. Em 2013 foram contabilizados um total de 173.462 atendimentos/ano.

O cálculo amostral foi realizado pela técnica probabilística aleatória levando em consideração a significância de $95 \%$ e erro de $5 \%$ e chegou-se 
a uma amostra de no mínimo 384 prontuários. A população pesquisada correspondeu aos usuários atendidos nos dois PA do município. Foi realizada uma amostra estratificada proporcional ao número de atendimentos entre os meses de janeiro a dezembro de 2013. Para finalizar, o processo de amostragem e aleatorização, dentro de cada mês, a seleção interna aos estratos obedeceu à amostragem aleatória simples

A coleta dos dados nos prontuários ocorreu em maio e junho de 2014. Após a seleção dos prontuários, foram excluídos 18 por se tratar de atendimentos ao mesmo usuário, sendo mantido o prontuário referente ao primeiro atendimento realizado no ano para este usuário. Também foram excluídos 13 prontuários por apresentarem incompletude de dados. Assim, realizou-se novo sorteio aleatório para inclusão de 31 prontuários a fim de completar a amostra de 384 .

Os atendimentos do PA foram categorizados em dois grupos: apropriados ou inapropriados. Para a classificação do atendimento do PA como apropriado, pelo menos um dos quatro critérios seguintes deveria ser contemplado, baseado em revisão sistemática sobre o tema ${ }^{6}$ : a) presença de queixa urgente/emergente, em que o usuário exigiu atenção imediata, com risco de morte ou lesões funcionais, de acordo com o relatório de atendimento profissional (médico e de Enfermagem). A classificação de risco segundo Manchester auxiliou na definição do caso, considerado uso apropriado o risco vermelho, laranja ou amarelo; b) sinais vitais fora dos limites da normalidade, de acordo com a faixa etária, relacionados com a queixa principal; c) conduta profissional adotada relacionada com a queixa principal (observação por mais de 12 horas ou hospitalização); e d) necessidade de exames de diagnóstico imediato ou tratamento especial, como medicação intravenosa, oxigênio etc. Caso o atendimento não contemplasse pelo menos um dos critérios acima descritos, foi classificado como inapropriado.

A análise dos prontuários foi realizada por dois pesquisadores de forma independente com posterior discussão de cada prontuário. Frente às divergências quanto à classificação de uso apropriado ou não, foram consultados experts na área (especialistas em urgência e emergência) para reavaliação do prontuário e definição da classificação final.

Foram coletadas as seguintes variáveis dos prontuários: sexo, data, horário e dia da semana do atendimento, faixa etária, existência de unidade de APS de referência para o usuário, diagnóstico do atendimento no PA (de acordo com a Décima Revisão da Classificação Internacional de Doenças e de Problemas Relacionados à Saúde - CID-10), entre maio e junho de 2014. As informações foram tabuladas no Programa Microsoft Office Excel ${ }^{\circledR} 2013$ para Windows ${ }^{\circledR}$.

A partir do diagnóstico descrito no prontuário foi realizada consulta à Lista Brasileira de Condições Sensíveis à Atenção Primária ${ }^{13}$ para caracterizar a variável como sendo ou não uma CSAP. Por meio da análise da data, horário e dia da semana do atendimento, foi possível categorizar se a APS estava aberta ou fechada, considerando que no município pesquisado as unidades de APS funcionavam de segunda a sexta-feira, exceto feriados, das $8 \mathrm{~h}$ às $12 \mathrm{~h}$ e das $13 \mathrm{~h}$ às $17 \mathrm{~h}$. Quanto a cobertura de APS, foi solicitado junto à Secretaria Municipal de Saúde o mapa de cobertura do município, e a partir do local de residência do usuário, determinou-se se ele pertencia à área coberta ou não.

As variáveis foram descritas com frequências e percentuais. A associação entre o uso inapropriado do atendimento no PA segundo as variáveis independentes foi avaliada pelo teste de Quiquadrado. A análise multivariada foi por meio de modelos de regressão logística utilizando o modelo stepwise forward, que estimaram o Odss Ratio (OR) e respectivos intervalos de confiança (IC). Foram incluídas no modelo as variáveis com $\mathrm{p}<0,20$ na análise bivariada. Foram mantidas no modelo final as variáveis que permaneceram significativas $(\mathrm{p}<0,05)$ ou que ajustaram o modelo. A adequação dos modelos finais foi verificada a partir dos testes de deviance, Hosmer-Lemeshow, a colinearidade das variáveis foi testada com o fator de inflação da variância (VIF) e as análises estatísticas realizadas com o software Stata versão 12 .

A pesquisa foi aprovada pelo Comitê de Ética em Pesquisa da Universidade Estadual do Centro-Oeste.

\section{Resultados}

Em 2013 foram realizados 173.462 atendimentos nas duas unidades de PA do município investigado, com uma média de 475 atendimentos/ dia. Dos 384 atendimentos incluídos no estudo, a proporção do uso inapropriado do PA foi de 73,4\%, (Tabela 1).

Quanto à caracterização dos atendimentos, verificou-se que a maioria dos atendimentos ocorreram de segunda à sexta-feira $(74,2 \%)$, em momentos que as Unidades Básicas de Saúde 
Tabela 1. Caracterização dos atendimentos em Unidades de Pronto Atendimento. Guarapuava, Paraná, Brasil, 2013 ( $\mathrm{n}=384)$.

\begin{tabular}{|c|c|c|}
\hline Características & $\mathbf{n}$ & $\%$ \\
\hline \multicolumn{3}{|l|}{ Uso inapropriado do $\mathrm{PA}^{1}$} \\
\hline Sim & 282 & 73,4 \\
\hline Não & 102 & 26,6 \\
\hline \multicolumn{3}{|l|}{$\mathrm{CSAP}^{2}$} \\
\hline Sim & 196 & 51,0 \\
\hline Não & 188 & 49,0 \\
\hline \multicolumn{3}{|l|}{ Área descoberta pela APS 3} \\
\hline Sim & 50 & 13,0 \\
\hline Não & 334 & 87,0 \\
\hline \multicolumn{3}{|l|}{ Período } \\
\hline $1^{\circ}$ trimestre & 95 & 24,7 \\
\hline $2^{\circ}$ trimestre & 93 & 24,2 \\
\hline $3^{\circ}$ trimestre & 97 & 25,3 \\
\hline $4^{\circ}$ trimestre & 99 & 25,8 \\
\hline \multicolumn{3}{|l|}{ Horário atendimento } \\
\hline $8 \mathrm{~h} 00 \mathrm{~min}$ às $16 \mathrm{~h} 59 \mathrm{~min}$ & 196 & 51,0 \\
\hline $17 \mathrm{~h} 00 \mathrm{~min}$ às $07 \mathrm{~h} 59 \mathrm{~min}$ & 188 & 49,0 \\
\hline \multicolumn{3}{|l|}{ Dia de atendimento } \\
\hline Dias úteis da semana & 285 & 74,2 \\
\hline Final de semana & 99 & 25,8 \\
\hline \multicolumn{3}{|l|}{$\mathrm{UBS}^{4}$ aberta } \\
\hline Sim & 143 & 37,2 \\
\hline Não & 241 & 62,8 \\
\hline \multicolumn{3}{|l|}{ Idade } \\
\hline 0 a 11 anos & 73 & 19,0 \\
\hline 12 a 17 anos & 34 & 8,9 \\
\hline 18 a 39 anos & 117 & 30,5 \\
\hline 40 a 59 anos & 96 & 25,0 \\
\hline 60 anos e mais & 64 & 16,7 \\
\hline \multicolumn{3}{|l|}{ Diagnóstico principal do atendimento ${ }^{5}$} \\
\hline Doenças do aparelho respiratório & 86 & 22,4 \\
\hline Causas mal definidas & 80 & 20,8 \\
\hline Lesões e envenenamentos & 39 & 10,2 \\
\hline Doenças do sistema osteomuscular & 31 & 8,1 \\
\hline $\begin{array}{l}\text { Fatores que influenciam o estado de } \\
\text { saúde }\end{array}$ & 24 & 6,3 \\
\hline Doenças do aparelho circulatório & 23 & 6,0 \\
\hline Doenças infecciosas e parasitárias & 20 & 5,2 \\
\hline Transtornos mentais e comportamentais & 15 & 3,9 \\
\hline Doenças do sistema nervoso & 13 & 3,4 \\
\hline Doenças do aparelho digestivo & 13 & 3,4 \\
\hline Doenças do aparelho geniturinário & 13 & 3,4 \\
\hline Doenças da pele e do tecido subcutâneo & 6 & 1,6 \\
\hline Causas externas & 6 & 1,6 \\
\hline Doenças do ouvido e da apófise & 4 & 1,0 \\
\hline
\end{tabular}

Demais capítulos da CID-10 $11 \quad 2,9$

Nota: ${ }^{1}$ Pronto atendimento, ${ }^{2}$ Condições sensíveis à Atenção Primária, ${ }^{3}$ Atenção Primária à Saúde, ${ }^{4}$ Unidade Básica de Saúde, ${ }^{5}$ Capítulos da CID-10.

Fonte: Dados da pesquisa.
(UBS) estavam fechadas $(62,8 \%)$, e para pessoas residentes em áreas com cobertura pela APS (87\%). A idade predominante foi para pessoas entre 18 e 39 anos (30,5\%). Dos agravos atendidos, $51 \%$ eram CSAP, e conforme os capítulos da CID-10, as principais doenças foram: doenças do aparelho respiratório $(22,4 \%)$, sintomas, sinais e achados anormais de exames clínicos e de laboratório, não classificados em outra parte (20,8\%) e lesões, envenenamentos e algumas outras consequências de causas externas (10,2\%) (Tabela 1).

A análise bivariada demonstrou que CSAP $(\mathrm{p}<0,001)$, área descoberta pela APS $(\mathrm{p}=0,009)$, primeiro trimestre do ano $(\mathrm{p}=0,013)$, faixa etária de 0 a 11 anos $(\mathrm{p}=0,006)$ e de 12 a 17 anos ( $\mathrm{p}=0,159)$, e os diagnósticos principais: doenças do aparelho respiratório $(\mathrm{p}<0,001)$, lesões e envenenamentos $(p<0,001)$ e causas externas $(\mathrm{p}=0,015)$, foram associadas ao uso do PA (Tabela 2).

A análise de regressão logística, mostrou que o uso inapropriado de PA foi associado a pessoas que foram atendidas por uma CSAP $(\mathrm{OR}=2,55)$. Além disso, esteve associado àqueles residentes em áreas sem cobertura pela APS $(\mathrm{OR}=2,30)$, pessoas com idade entre 0 e 11 anos $(\mathrm{OR}=4,37)$, e entre 12 e 17 anos $(\mathrm{OR}=3,67)$ doenças do aparelho respiratório $(\mathrm{OR}=3,82)$, e doenças do sistema osteomuscular $(\mathrm{OR}=3,19)$, e a efeitos protetores para $\mathrm{o}$ atendimento no primeiro trimestre do ano $(\mathrm{OR}=0,40)$ e lesões e envenenamentos $(\mathrm{OR}=0,11)$ (Tabela 3$)$.

\section{Discussão}

Por mais de duas décadas estuda-se sobre o uso inapropriado de PA, uma preocupação mundial discutida em vários países com diferentes contextos de saúde ${ }^{6,10,14}$. Nesse estudo, se observou elevada proporção de uso inapropriado dos serviços de PA, o que corrobora com a literatura ${ }^{5,15,16}$.

Deve-se considerar que as proporções encontradas nos estudos podem estar relacionadas especialmente aos métodos utilizados para determinar as situações de uso inapropriado ${ }^{6}$ que se caracterizam por situações não urgentes ou emergentes atendidas em serviços de $\mathrm{PA}^{1,2,5}$, ou seja, não se constituem em quadros agudos/ agudizados de natureza clínica, cirúrgica ou traumática9.

Apesar de definidos na literatura, ainda há controvérsias sobre os critérios para classificação de casos de uso inapropriado do $\mathrm{PA}^{10}$. Neste estudo, adotaram-se os critérios de gravidade para 
Tabela 2. Associação bivariada entre uso inapropriado do Pronto Atendimento e variáveis independentes. Guarapuava, Paraná, Brasil, 2013 ( $\mathrm{n}=384)$.

\begin{tabular}{|c|c|c|c|c|c|c|}
\hline \multirow{2}{*}{ Variáveis } & \multicolumn{2}{|c|}{ Apropriado } & \multicolumn{2}{|c|}{ Inapropriado } & \multirow{2}{*}{ OR } & \multirow{2}{*}{ p-valor } \\
\hline & n & $\%$ & $\mathrm{n}$ & $\%$ & & \\
\hline \multicolumn{7}{|l|}{$\mathrm{CSAP}^{2}$} \\
\hline Sim & 24 & 23,5 & 172 & 61 & 5,08 & $<0,001$ \\
\hline Não & 78 & 76,5 & 110 & 39 & ref $^{3}$ & \\
\hline \multicolumn{7}{|l|}{ Área descoberta pela $\mathrm{APS}^{4}$} \\
\hline Sim & 21 & 20,6 & 29 & 10,3 & ref. & \\
\hline Não & 81 & 79,4 & 253 & 89,7 & 2,26 & 0,009 \\
\hline \multicolumn{7}{|l|}{ Sexo } \\
\hline Masculino & 51 & 50 & 123 & 43,6 & ref. & \\
\hline Feminino & 51 & 50 & 159 & 56,4 & 1,29 & 0,268 \\
\hline \multicolumn{7}{|l|}{ Período do ano } \\
\hline $1^{\circ}$ trimestre & 41 & 40,2 & 54 & 19,1 & 0,45 & 0,013 \\
\hline $2^{\circ}$ trimestre & 18 & 17,6 & 79 & 28 & 1,52 & 0,230 \\
\hline $3^{\circ}$ trimestre & 19 & 18,6 & 80 & 28,4 & 1,46 & 0,273 \\
\hline $4^{\circ}$ trimestre & 24 & 23,5 & 69 & 24,5 & ref. & \\
\hline \multicolumn{7}{|l|}{ Horário atendimento } \\
\hline $8: 00$ às $16: 59$ horas & 53 & 52 & 143 & 50,7 & 0,95 & 0,828 \\
\hline $17: 00$ às $07: 59$ horas & 49 & 48 & 139 & 49,3 & ref. & \\
\hline \multicolumn{7}{|l|}{ Dia de atendimento } \\
\hline Dias da semana & 28 & 27,5 & 71 & 25,2 & 0,88 & 0,653 \\
\hline Final de semana & 74 & 72,5 & 211 & 74,8 & ref. & \\
\hline \multicolumn{7}{|l|}{ APS $^{4}$ aberta } \\
\hline Sim & 40 & 39,2 & 103 & 36,5 & 0,89 & 0,630 \\
\hline Não & 62 & 60,8 & 179 & 63,5 & ref. & \\
\hline \multicolumn{7}{|l|}{ Faixa etária } \\
\hline 0 a 11 anos & 10 & 9,8 & 63 & 22,3 & 3,30 & 0,006 \\
\hline 12 a 17 anos & 7 & 6,9 & 27 & 9,6 & 2,02 & 0,159 \\
\hline 18 a 39 anos & 35 & 34,3 & 82 & 29,1 & 1,22 & 0,537 \\
\hline 40 a 59 anos & 28 & 27,5 & 68 & 24,1 & 1,27 & 0,487 \\
\hline+ de 60 anos & 22 & 21,6 & 42 & 14,9 & ref. & \\
\hline \multicolumn{7}{|l|}{ Diagnóstico principal $^{5}$} \\
\hline Doenças infecciosas e parasitárias & 1 & 5 & 19 & 95 & 7,26 & 0,054 \\
\hline Doenças endócrinas, nutricionais e metabólicas & 2 & 66,7 & 1 & 33,3 & 0,14 & 0,161 \\
\hline Transtornos mentais e comportamentais & 5 & 33,3 & 10 & 66,7 & 0,71 & 0,546 \\
\hline Doenças do sistema nervoso & 4 & 30,8 & 9 & 69,2 & 0,8 & 0,727 \\
\hline Doenças do aparelho circulatório & 6 & 26,1 & 17 & 73,9 & 1,02 & 0,958 \\
\hline Doenças do aparelho respiratório & 4 & 4,7 & 82 & 95,3 & 10,04 & $<0,001$ \\
\hline Doenças do aparelho digestivo & 3 & 23,1 & 10 & 76,9 & 1,21 & 0,773 \\
\hline Doenças da pele e do tecido subcutâneo & 2 & 33,3 & 4 & 66,7 & 0,71 & 0,706 \\
\hline Doenças do sistema osteomuscular & 5 & 16,1 & 26 & 83,9 & 1,97 & 0,177 \\
\hline Doenças do aparelho geniturinário & 6 & 46,2 & 7 & 53,8 & 0,4 & 0,114 \\
\hline Causas mal definidas & 22 & 27,5 & 58 & 72,5 & 0,94 & 0,831 \\
\hline Lesões e envenenamentos & 31 & 79,5 & 8 & 20,5 & 0,06 & $<0,001$ \\
\hline Causas externas & 5 & 83,3 & 1 & 16,7 & 0,06 & 0,015 \\
\hline Fatores que influenciam o estado de saúde & 5 & 20,8 & 19 & 79,2 & 1,4 & 0,513 \\
\hline
\end{tabular}

Fonte: Dados da pesquisa. 
Tabela 3. Fatores independentes associados ao uso inapropriado do Pronto Atendimento. Guarapuava, Paraná, Brasil, 2013 ( $\mathrm{n}=384)$.

\begin{tabular}{lcr}
\hline \multicolumn{1}{c}{ Variáveis } & OR & \multicolumn{1}{c}{ IC $^{\mathbf{l}}$} \\
\hline CSAP $^{2}$ & 2,55 & 1,$33 ; 4,87$ \\
Área descoberta pela APS & 2,30 & 1,$11 ; 4,78$ \\
0 a 11 anos & 4,37 & 1,$46 ; 13,02$ \\
12 a 17 anos & 3,67 & 1,$07 ; 12,51$ \\
Doenças do aparelho respiratório & 3,79 & 1,$21 ; 11,85$ \\
Lesões e envenenamentos & 0,11 & 0,$04 ; 0,28$ \\
Doenças do sistema osteomuscular & 3,20 & 1,$10 ; 9,31$ \\
1 ${ }^{\text {o trimestre }}$ & 0,40 & 0,$19 ; 0,85$ \\
\hline${ }^{1}$ Intervalo de confiança, ${ }^{2}$ Condições sensíveis à Atenção \\
Primária, ${ }^{3}$ Atenção Primária à Saúde.
\end{tabular}

Fonte: Dados da pesquisa.

classificar a utilização inapropriada ou apropriada do PA, com base em rigorosos critérios difundidos na literatura ${ }^{6}$. Esse rigor é importante quando se considera a construção dos sistemas de saúde locais orientados pela APS, ao viabilizar a compreensão do papel de cada ponto de atenção, como em estudo realizado na França, que analisou os atendimentos em PA e constatou que situações não urgentes atendidas no PA poderiam ser gerenciadas por médicos generalistas na APS $^{10}$.

O presente estudo demonstrou que as pessoas atendidas por alguma CSAP tiveram maior chance de uso inapropriado do PA. Pesquisa em município do Paraná apontou que a presença de morbidade crônica e falta de médico nas UBS são fatores associados ao uso inapropriado do PA por idosos com condições ${ }^{17}$. Por outro lado, o aumento na densidade de clínicos gerais e a estruturação de rede assistencial apresenta impacto na redução de internações por CSAP ${ }^{18}$.

A agudização de uma CSAP é caracterizada como um caso de uso apropriado do PA, já que esse é estruturado para atender situações de urgência e emergência. Entretanto, tal ocorrência remete às falhas no manejo e controle dos referidos agravos no âmbito da APS, e que mostra sua fragilidade no controle das CSAP.

A APS deve ser constantemente avaliada a fim de incentivar melhorias para que seu papel seja cumprido. Se as CSAP forem atendidas de forma resolutiva na APS, o risco de agravo e posterior agudização, que leva à procura por atendimento em PA e/ou internação hospitalar será reduzido ${ }^{19}$. Além disso, quando se acessa de maneira frequente e não urgente um $\mathrm{PA}$, o usuário não tem continuidade no cuidado oferecido pela APS.
As CSAP são responsáveis pela maior parte das internações por condições clínicas, sendo que o investimento financeiro no Sistema Único de Saúde, expansão da ESF e do programa Mais Médicos tem impactos importantes na redução dessas internações ${ }^{20,21}$, estratégias essas de valorização e fortalecimento da APS.

Pode-se pressupor, conforme os resultados do presente estudo, que ao atender efetivamente as CSAP no âmbito da APS os benefícios serão não somente a redução das internações, mas também dos atendimentos nos outros níveis de atenção ${ }^{20}$, com diminuição dos custos e melhoria na qualidade da atenção à saúde ${ }^{2,22}$.

Uma das causas de ineficiência do sistema de saúde são solicitações inadequadas de serviços, uma vez que parcela significativa das despesas com a saúde é reservada para procedimentos considerados desnecessários. A intenção inicial do usuário em procurar um serviço de saúde pode diferir da ação que seria mais adequada em termos de organização do sistema, o que gera um custo inoportuno ${ }^{23}$. Cabe então uma reorganização dos serviços de saúde com valorização da APS, já que quando esta não oferta um serviço estruturado, resolutivo e em tempo oportuno, o usuário busca atendimento no serviço de saúde que melhor atende aos seus anseios.

Os resultados também evidenciaram que usuários de regiões sem cobertura pela APS têm maior chance de uso inapropriado de PA, e isto pode ser explicado pela falta de acesso regular aos cuidados primários, o que resulta na busca por serviços de PA para qualquer condição ou problema ${ }^{4}$, situação comum em países de baixa renda $^{14}$. A maioria dos usuários atendidos em serviços de PA poderia receber atendimento sem necessidade de recursos tecnológicos acurados, o que reduziria a superlotação dos serviços de emergência ${ }^{3}$.

Mesmo que a literatura sobre o impacto das ações da APS para a busca de setores de emergência seja escassa, existe interesse em intervenções que facilitem o uso adequado do PA. Estudos relataram que o empoderamento ou o cuidado centrado no usuário podem diminuir essa inadequação ${ }^{12}$. Nos Estados Unidos, distribuir materiais educativos com instruções sobre como lidar com problemas simples em seus domicílios e como decidir sobre quando buscar os serviços do PA são importantes para melhorar o fluxo de atendimento em $\mathrm{PA}^{24}$. O desenvolvimento de tecnologias da informação e comunicação podem ser ferramentas que fornecem informações aos usuários e foram capazes de auxiliar na tomada de decisões, 
como ferramentas de saúde móvel ( $m$-health) e plataformas online de aconselhamento médico ${ }^{23}$. Apesar desses esforços, as dificuldades na procura adequada pelos serviços de PA permanecem em ascensão em diferentes países?

No Brasil, a partir de 2009, iniciou-se a implantação das UPAs, as quais deveriam ser instaladas estrategicamente para configurar a rede de atenção às urgências, diferenciando-se dos PA, os quais produzem "consultas de emergência" 25 . Porém, foi um processo acelerado, sem concretização do propósito de organização da rede, e reproduziu o modelo tradicional de PA e pouco impactou positivamente para enfrentar os problemas de atenção às urgências ${ }^{25}$.

A superlotação do PA por causas passíveis de resolução na APS implica em subutilização desta, e sobrecarga do primeiro, tanto em termos de trabalho das equipes de urgência e emergência, quanto dos custos para o sistema de saúde ${ }^{10,22}$.

Além dos aspectos próprios do uso inapropriado do PA, deve-se destacar as questões específicas de estruturação e instrumentalização da APS. Um dos desafios enfrentados pelo sistema de saúde brasileiro é tornar a APS porta de entrada dos usuários, a qual torna possível a resolutividade da maioria dos agravos em saúde. A escassez de recursos decorrente do subfinanciamento e a falta de resolutividade sobrecarregam os outros pontos de atenção com influência direta na qualidade do serviço prestado ${ }^{15,17,26}$.

A APS tem sido reconhecida como um componente central dos sistemas de saúde eficazes desde o início do século XX. No entanto, apesar do notável progresso, ainda existe uma grande lacuna entre o que os indivíduos e as comunidades necessitam, e a qualidade dos cuidados pres$\operatorname{tados}^{27}$.

No Brasil existem falhas consideráveis na forma como os cuidados primários são organizados, financiados e prestados, e tais questões estão associadas a problemas no desempenho do sistema de saúde, incluindo o recebimento de assistência médica em tempo oportuno e eficaz ${ }^{28}$. A APS ainda tem altos níveis de barreiras organizacionais, falta de continuidade dos cuidados, especialmente por dificuldades na fixação de profissionais nesse ponto de atenção. Outro problema importante são falhas na coordenação dos cuidados, que reflete o desafio de incorporar com sucesso a prevenção secundária e os serviços avançados de diagnóstico na $\mathrm{APS}^{28}$.

O estudo encontrou associação entre duas faixas etárias, entre 0 a 11 anos e 12 a 17 anos, com uso inapropriado do PA, e corrobora com achados de outros estudos ${ }^{3,4,15,16}$. Na Austrália, o uso dos departamentos de emergência por situações não caracterizadas como urgência diminui proporcionalmente ao aumento da idade ${ }^{3}$. Os idosos têm maior acompanhamento das condições crônicas pela APS, o que diminui as chances de uso inapropriado do $\mathrm{PA}^{15}$.

A busca pelo atendimento a crianças e adolescentes no PA por condições não urgentes envolve a avaliação do cuidador/responsável sobre os benefícios e desvantagens de frequentar um PA em comparação ao atendimento em APS, além dessa avaliação ser influenciada pela percepção da gravidade da doença e disponibilidade do serviço no momento que o usuário considera necessário ${ }^{8}$.

Existem dificuldades de acesso à APS por adolescentes devido a problemas na acessibilidade geográfica e organizacional ${ }^{29}$, sendo que é premente a priorização de serviços amigáveis para eles, como o horário de funcionamento que permite que os jovens saiam em horário de aula $\mathrm{e}$ profissionais com habilidades para trabalhar com este público. Em geral, esses serviços devem ser prestados de forma disponível para os adolescentes, mas também aceitáveis e acessíveis ${ }^{30}$.

Como forma de otimizar o itinerário terapêutico a ser percorrido, é essencial melhorar o conhecimento dos cuidadores sobre questões de saúde infantil ${ }^{8}$, bem como sobre o fluxo dos atendimentos urgentes no sistema de saúde, além de aprimorar aspectos organizacionais do sistema de saúde, especialmente pela ampliação da acessibilidade socio-organizacional, como implantação de horário de atendimento estendido na APS.

$\mathrm{O}$ uso inapropriado também foi associado às doenças do sistema respiratório, comuns na região do município estudado, especialmente pelo clima frio. Houve associação também com as doenças do sistema osteomuscular, agravos predominantes em outros estudos sobre o tema ${ }^{1,16} \mathrm{e}$ comuns entre trabalhadores, em geral homens, da indústria madeireira do município.

Tanto as doenças do sistema respiratório como osteomuscular constituem-se em condições passíveis de prevenção no âmbito da $\mathrm{APS}^{16}$, por meio de estratégias para enfrentamento de doenças e agravos, com tratamento precoce e articulação intersetorial para intervenção em saúde do trabalhador.

Já as lesões e envenenamentos apresentaramse como fator de proteção para uso inapropriado, o que pode ser explicado pelo fato de se constituírem em condições agudas, as quais estão adequadamente referenciadas para atendimento em unidade de $\mathrm{PA}^{6}$. 
$\mathrm{O}$ atendimento no primeiro trimestre do ano teve associação negativa com uso inapropriado possivelmente por uma questão comportamental, em que geralmente as pessoas, em especial crianças, estão em período de férias, portanto longe do contato em ambientes fechados com outras pessoas, o que minimiza a ocorrência de doenças, especialmente respiratórias. Além disso, o município possui um clima de verão ameno, com menor frequência dos agravos próprios de clima de verão intenso.

O presente estudo apresentou algumas limitações, como coleta de dados somente em prontuários, limitando a associação aos dados disponíveis, falta de padronização entre diferentes estudos sobre uso inapropriado de PA e por se tratar de um estudo transversal em que não foi possível estabelecer relações de causa e efeito. Apesar dessas limitações, foi possível construir maior entendimento sobre o uso inapropriado de PA e seus fatores associados.

\section{Conclusões}

O uso inapropriado do PA é capaz de gerar um desajuste do sistema de saúde, ao torná-lo de- sorganizado e replicante do modelo hegemônico curativista, faz-se necessário conhecer os fatores que influenciam nesse uso inapropriado. Esse conhecimento poderia esclarecer condições e situações que podem ser melhoradas e modificadas na rede de atenção à saúde para assistir aos usuários com maior qualidade, resolutividade e menores custos para o sistema.

Neste estudo os resultados indicaram uma alta proporção de uso inapropriado de PA, associado às CSAP e à falta de cobertura da APS, demonstrando que a fragilidade da APS impacta na sobrecarga de outros pontos de atenção. Além disso, o uso inapropriado foi associado às faixas etárias de 0 a 11 anos e de 12 a 17 anos, acometidos por doenças do aparelho respiratório e do sistema osteomuscular. Atendimentos realizados no primeiro trimestre do ano e por lesões e envenenamentos apresentaram-se como proteção para o uso inapropriado do PA.

Enfatiza-se a necessidade de fortalecimento da APS para redução do uso inapropriado do PA. Presume-se que o redirecionamento dos atendimentos não urgentes para a APS seja uma meta de gestão desejável, a fim de que o pronto atendimento concentre esforços em usuários em situações graves.

\section{Colaboradores}

T Baratieri, MH Lentsck contribuiu na concepção do projeto, análise e interpretação dos dados, redação do trabalho, revisão crítica do conteúdo, e aprovação da versão final a ser publicada. LP Corona contribuiu com a análise e interpretação dos dados, redação do trabalho, e aprovação da versão final a ser publicada. KP Almeida contribuiu na concepção do projeto, análise dos dados, redação do trabalho e aprovação da versão final a ser publicada. ACGC Kluthcovsky contribuiu na análise e interpretação dos dados, revisão crítica do conteúdo e aprovação da versão final a ser publicada. S Natal contribuiu na análise e interpretação dos dados, revisão crítica do conteúdo e aprovação da versão final a ser publicada. 


\section{Referências}

1. Unwin M, Nurs GD, Kinsman L. Rigby S, Nurs GC. Why are we waiting? Patients' perspectives for accessing emergency department services with non-urgent complaints. Int EmergNurs 2016 [cited 2018 Mar 15]. 29:3-8. Available from: https://www.sciencedirect. com/science/article/pii/S1755599X16300994?via\%3Dihub

2. Cremonesi P, Bella E, Montefiori M, Persico L. The Robustness and Effectiveness of the Triage System at Times of Overcrowding and the Extra Costs due to Inappropriate Use of Emergency Departments. Appl Health Econ Health Policy 2015 [cited 2018 Mar 20]. 13(5):507-514. Available from: https://www.ncbi.nlm. nih.gov/pubmed/25854901

3. Freed G, Gafforini S, Carson N. Age-related variation in primary care type presentations to emergency departments. Aust Fam Physician 2015 [cited 2018 Fev 20]; 44(8):584-588. Available from: https://www.ncbi. nlm.nih.gov/pubmed/26510148

4. Parry YK, Ullah S, Raftos J, Willis E. Deprivation and its impact on non-urgent Paediatric Emergency Department use: are Nurse Practitioners the answer? J Adv Nurs 2016 [cited 2018 Mar 14]; 72(1):99-106. Available from: https://www.ncbi.nlm.nih.gov/pub $\operatorname{med} / 26383900$

5. Alyasin A, Douglas C. Reasons for non-urgent presentations to the emergency department in Saudi Arabia. Int Emerg Nurs 2014 [cited 2018 Mar 12]; 22(4):220225. Available from: https://www.ncbi.nlm.nih.gov/ pubmed/24703789

6. Carret MLV, Fassa AG, Domingues MR. Inappropriate use of emergency services: a systematic review of prevalence and associated factors. Cad Saude Publica 2009 [cited 2013 Mar 30];25(1):7-28. Available from: https://www.ncbi.nlm.nih.gov/pubmed/19180283

7. Coster JE, Turner JK, Bradbury D, Cantrell A. Why do people choose emergency and urgent care services? a rapid review utilizing a systematic literature search and narrative synthesis. Acad Emerg Med 2017 [cited 2018 Mar 13]; 24(9): 1137-1149. Available from: https://www.ncbi.nlm.nih.gov/pmc/articles/PMC 5599959/

8. Hui Jade Kua P, Wu L, Tessa Ong E-L, Ying Lim Z, Luther Yiew J, Hui Michelle Thia X, et al. Understanding decisions leading to nonurgent visits to the paediatric emergency department: caregivers' perspectives. Singapore Med J [Internet]. 2016 [cited 2019 May 24]; 57(6):314-319. Available from: https://www.ncbi.nlm. nih.gov/pmc/articles/PMC4971450/pdf/SMJ-57-314. pdf

9. Brasil. Ministério da Saúde. Portaria n. 10, de 3 de janeiro de 2017. Redefine as diretrizes de modelo assistencial e financiamento de UPA 24h de Pronto Atendimento como Componente da Rede de Atenção às Urgências, no âmbito do Sistema Único de Saúde. Brasília: MS;2017 [acessado 2018 fev 5]. Disponível em: http:// http://bvsms.saude.gov.br/bvs/saudelegis/ gm/2017/prt0010_03_01_2017.html

10. Colineaux H, Querrec FL, Pourcel L, Gallart JC, Azéma O, Lang T, Kelly-Irving M, Charpentier S, Lamy S. Is the use of emergency departments socially patterned? Int J Public Health 2018 [cited 2018 Ago 02]; 63(3): 397-407. Available from: https://www.ncbi. nlm.nih.gov/pubmed/29332173
11. Chapman B, Turnbull T. Health professionals' perceptions of inappropriate use of A\&E services. $\mathrm{Br} J$ Nurs 2016 [cited 2018 Mar 15]; 25(9):476-483. Available from: https://www.ncbi.nlm.nih.gov/pubmed/27 172488

12. Hudon C, Sanche S, Haggerty JL. Personal characteristics and experience of primary care predicting frequent use of emergency department: a prospective cohort study. PLoS One 2016 [cited 2019 May 24];11(6):e0157489. Available from: http://dx.doi.org /10.5061/dryad

13. Brasil. Ministério da Saúde. Portaria n. 221, de 17 de abril de 2018. Dispõe sobre as internações por Condições Sensíveis à Atenção Primária. Brasília; 2018 [acessado 2018 fev 5]. Disponível em: http:// bvsms.saude.gov.br/bvs/saudelegis/sas/2008/ prt0221_17_04_2008.html

14. Read JG, Varughese S, Cameron PA. Determinants of non-urgent Emergency Department attendance among females in Qatar. Qatar Med J. 2014 [cited 2018 Abr 21]; 2014(2):98-105. Available from: https:// www.ncbi.nlm.nih.gov/pubmed/25745599

15. Machado GVC, Oliveira FLP, Barbosa HAL, Giatti L, Bonolo PF. Fatores associados à utilização de um serviço de urgência/emergência. Cad Saude Colet 2015 [acessado 2018 abr 18]; 23(4):416-424. Disponível em: http://www.scielo.br/scielo.php?pid=S1414-462X 2015000400416\&script $=$ sci_abstract\&tlng $=\mathrm{pt}$

16. Garcia VM, Reis RK. Adequação da demanda e perfil de morbidade atendida em uma unidade não hospitalar de urgência e emergência. Cien Cuid Saude 2014 [acessado 2018 abr 18]. 13(4):665-673. Disponível em: http://dx.doi.org/10.4025/cienccuidsaude. v13i4.19127

17. Rêgo AS, Rissardo LK, Scolari GAS, Sanches RCN, Carreira L, Radovanovic CAT. Fatores associados ao atendimento a idosos por condições sensíveis à Atenção Primária à Saúde. Rev Bras Geriatr Gerontol 2017 [acessado 2018 fev 18]; 20(6):778-789. Disponível em: http://www.scielo.br/scielo.php?pid=S 1809-98232017000600773\&script=sci_abstract\&tlng=pt.

18. Burgdorf F, Sundmacher L. Potentially Avoidable Hospital Admissions in Germany An Analysis of Factors Influencing Rates of Ambulatory Care Sensitive Hospitalizations. Dtsch Arztebl Int 2014 [cited 2019 May 24];111(13):215-223. Available from: https:// www.ncbi.nlm.nih.gov/pmc/articles/PMC3991158/ pdf/Dtsch_Arztebl_Int-111-0215.pdf

19. Costa JSD, Pattussi MP, Morimoto T, Arruda JS, Bratkowski GR, Sopelsa M, Fritzen JS, Canto VAF, Marques MC. Tendência das internações por condição sensível à atenção primária e fatores associados em Porto Alegre, RS, Brasil. Cien Saude Colet 2016 [acessado 2018 mar 12]; 21(4):1289-1296. Disponível em: http://www.scielo.br/scielo.php?pid=S1413-8123201 6000401289 \&script $=$ sci_abstract\&tlng $=$ pt

20. Gonçalves MR, Hauser L, Prestes IV, Schmidt MI, Duncan BB, Harzheim E. Primary health care quality and hospitalizations for ambulatory care sensitive conditions in the public health system in Porto Alegre. Brazil Fam Pract 2016 [cited 2018 Apr 21]; 33(3):238242. Available from: https://www.ncbi.nlm.nih.gov/ pubmed/26124441. 
21. Paim Brasil V, Soares Dias da Costa J. Hospitalizações por condições sensíveis à atenção primária em Florianópolis, Santa Catarina - estudo ecológico de 2001 a 2011. Epidemiol Serv Saude 2016;25(1):10-11.

22. Rudge GM, Mohamed MA, Fillingham SC, Girling A, Sidhu K, Stevens AJ. The combined influence of distance and neighbourhood deprivation on Emergency Department attendance in a large English population: a retrospective database study. Plos One. 2013 [cited 2018 Apr 21]; 8(7):e67943. Available from: https:// www.ncbi.nlm.nih.gov/pubmed/23874473

23. Guimarães DS, Soares EJ, Júnior GF, Medeiros DD. Attributes and circumstances that induce inappropriate health services demand: a study of the health sector in Brazil. BMC Health Serv Res 2015; 15:65 [cited 2018 Apr 15]. Available from: https://www.ncbi.nlm. nih.gov/pmc/articles/PMC4339295/

24. Yoffe SJ, Moore RW, Gibson JO, Dadfar NM, McKay $\mathrm{RL}$, McClellan DA et al. A reduction in emergency department use by children from a parent educational intervention. Fam Med 2011 [cited 2018 Mar 21]. Available from: https://www.ncbi.nlm.nih.gov/ pubmed/21305425/

25. Konder MT, O’Dwyer G. As Unidades de Pronto-Atendimento na Política Nacional de Atenção às Urgências. Physis. 2015 [acessado 2018 abr 19]; 25(2):525-545. Disponível em: http://www.scielo. br/scielo.php?script $=$ sci_arttext $\&$ pid $=$ S0103-733 $12015000200525 \& \operatorname{lng}=p t \& t \operatorname{lng}=p t$

26. Oliveira AMS, Menezes TMO. The nurse in the elderly care of the family health strategy: meanings of the experience. Rev Enferm UERJ 2014 [cited 2018 Apr 21]; 22(4):513-518. Available from: http://www.e-publicacoes.uerj.br/index.php/enfermagemuerj/article/ view/15373/11629

27. Bitton A, Ratcliffe HL, Veillard JH, Kress DH, Barkley S, Kimball M, Secci F, Wong E, Basu L, Taylor C, Bayona J, Wang H, Lagomarsino G, Hirshhorn LR. Primary Health Care as a Foundation for Strengthening Health Systems in Low- and Middle-Income Countries. J Gen Intern Med 2016 [cited 2019 Abr 23];32(5):566-571. Available from: https://link.springer.com/content/pdf/10.1007\%2Fs11606-016-3898-5.pdf
28. Macinko J, Guanais FC, Mullachery P, Jimenez G. Gaps in primary care and health system performance in six latin American and Caribbean countries. Health Aff 2016;35(8):1513-1521.

29. Martins MMF, Aquino R, Pamponet ML, Pinto Junior EP, Amorim LDAF. Acesso aos serviços de atenção primária à saúde por adolescentes e jovens em um município do Estado da Bahia, Brasil. Cad Saude Publica 2019 [acessado 31 maio 2019]; 35(1):e00044718. Disponível em: https://cidades.ibge.gov.br/brasil/ba/ camaca

30. James S, Pisa PT, Imrie J, Beery MP, Martin C, Skosana $\mathrm{C}$, et al. Assessment of adolescent and youth friendly services in primary healthcare facilities in two provinces in South Africa. BMC Health Serv Res 2018 [cited 2019 May 31]; 18(1):809. Available at: http://www. ncbi.nlm.nih.gov/pubmed/30348166

Artigo apresentado em 13/12/2018

Aprovado em 09/08/2019

Versão final apresentada em 11/08/2019

Editores-chefes: Romeu Gomes, Antônio Augusto Moura da Silva 